\title{
Narration(s) : en deçà et au-delà
}

In: Communications, 38, 1983. pp. 192-212.

Citer ce document / Cite this document :

Jost François. Narration(s) : en deçà et au-delà. In: Communications, 38, 1983. pp. 192-212.

doi : $10.3406 /$ comm.1983.1573

http://www.persee.fr/web/revues/home/prescript/article/comm_0588-8018_1983_num_38_1_1573 


\section{François Jost}

\section{Narration(s) : en deçà et au-delà}

Comment l'image signifie?

Comment limage raconte?

On me concédera, sans doute, que ces deux questions ne sont pas synonymes. Et pourtant : n'est-ce pas pour les avoir trop longtemps confondues que la théorie du cinéma s'est peu préoccupée, pour l'instant, des problèmes de narratologie?

En effet, jusqu'à ces dernières années, le récit cinématographique a généralement été envisagé sous l'angle de la signification. Ainsi, la sémiologie s'est attachée d'abord à comprendre comment le cinéma exprime les différentes relations temporelles (succession, précession), logiques (opposition, causalité, etc.), n'abordant les catégories du récit que par la bande. Poursuivant simultanément ses investigations sur le langage et sur le récit cinématographique, cette jeune discipline n'a travaillé les concepts narratologiques que pour autant qu'ils éclairaient, du même coup, tel ou tel aspect du matériau audiovisuel. Combien paradoxale pourrait paraître cette démarche à un théoricien de la littérature! Imagine-t-on un narratologue qui, pour analyser le texte romanesque, déciderait de reconstruire l'édifice de la langue?

En fait, le parallèle n'est pas très légitime et le paradoxe n'est qu'apparent. Si le théoricien de la littérature peut se dispenser d'étudier la nature du signe linguistique pour comprendre le fonctionnement d'un roman, il n'empêche que la langue - objet familier qu'il fréquente depuis son plus jeune âge - reste son plus fidèle indicateur pour repérer les catégories narratives. Il suffit, pour s'en convaincre, de considérer le récit sous l'aspect de la narration ${ }^{1}$ : bien que, dans le roman, ce problème ne se réduise pas à un simple emploi de personnes grammaticales (et suppose une attitude narrative), les pronoms, les déictiques, les signes de ponctuation sont des indicateurs irremplaçables pour repérer celui qui parle. Un simple adverbe (ici, aujourd'hui), même s'il est placé dans un texte à la troisième personne, renvoie forcément à un narrateur invisible, contemporain de la fiction qui s'élabore ${ }^{2}$. Ainsi, la langue, parce qu'elle prend forcément parti pour « le discours » ou pour "l'histoire " (selon la distinction de Benveniste) contraint le romancier à effectuer, même malgré lui ${ }^{3}$, un choix narratif que le lecteur repérera sans trop de mal. S'agissant de cinéma, les problèmes de narration sont 
singulièrement plus complexes pour des raisons qui tiennent, d'une part à la nature du récit cinématographique, d'autre part à son langage.

La première difficulté, en effet, c'est qu'il ne paraît guère plus possible de caractériser une narration cinématographique, qu'un récit unique. Partagé entre le visuel et le verbal, celui-ci risque toujours d'être écartelé entre deux sens contradictoires, même si, généralement, des stratégies sont mises en œuvre pour aboutir à un signifié global ${ }^{4}$. Notamment, l'utilisation de la voix off peut provoquer l'éclatement de l'instance narrative à l'intérieur d'un film. Dans ces conditions est-il possible, et souhaitable, de concevoir la narration selon un concept unitaire?

La seconde difficulté résulte des caractères sémiologiques de "l'énoncé iconique ». On pourrait la résumer dans ce paradoxe : je suis sûr que la suite d'images est susceptible de raconter et que le récit visuel existe ; et pourtant il n'est pas toujours possible de dire qui raconte. Existerait-il des récits sans narrateurs?

\section{LA NARRATION SANS VOIX.}

\section{Signifier et raconter.}

Derrière le problème de la narration filmique se cachent donc deux questions beaucoup plus larges, dont la sémiologie, semble-t-il, n'est pas encore venue à bout : comment l'image mouvante raconte-t-elle? Comment s'articulent mots et images à l'intérieur de ce texte complexe que constitue le film?

$\mathrm{Si}$, il y a quelques années, il importait de rapprocher le plan de l'énoncé (puisque d'aucuns l'identifiaient au mot), il semble qu'aujourd'hui, pour progresser dans l'analyse conceptuelle d'une narratologie proprement cinématographique, il faille au contraire creuser les différences séparant l'iconique et le linguistique. Retour à la case départ, donc : dans quelle mesure le plan ressemble-t-il à l'énoncé ? On peut d'abord envisager cette question sous l'angle de la signification. De ce point de vue, on dira, par exemple, que l'image d'une maison ne signifie pas une maison. mais "voici une maison " ". Si cette "traduction" intersémiotique, qui fait du plan un énoncé déictique, a le mérite d'écarter toute théorie qui le réduirait à un mot, elle n'est plus assez précise dès lors que l'on s'intéresse à l'image, non plus dans une perspective purement sémiologique, mais plutôt dans le but de contribuer à l'élaboration d'une narratologie cinématographique. En effet, des traits définitoires du langage cinématographique comme "la valeur déictique d'un plan » ou "la quantité indéfinie des informations qu'il est susceptible de véhiculer» ont une portée très différente à partir du 


\section{François Jost}

moment où l'on envisage, plus globalement, leurs effets narratifs à l'intérieur d'un film. Une narratologie cinématographique se doit donc de repartir des résultats de la sémiologie pour en tirer les conséquences par rapport au récit. Ainsi, on ne contestera pas que, du point de vue de la signification, l'image possède en propre, indépendamment de tout contexte, une valeur déictique qui renvoie l'iconique au référent, mais cette propriété devient insuffisante pour déterminer le sens diégétique d'un plan à l'intérieur d'un film. Il n'est plus possible alors de se contenter d'une analogie qui permettrait de décrire le plan comme un groupe d'énoncés : il faut aussi savoir quel type d'énoncé pourrait le caractériser. Dirai-je de l'image d'une maison qu'elle signifie "voici une maison " ou, au contraire, devrai-je dire qu'elle équivaut à "voici ma maison"? Les deux traductions sont loin d'être identiques : dans le premier cas, le plan n'a pas de locuteur déterminable (et on fait l'économie d'une partie du problème de l'énonciation), dans le second, elle est comme racontée par quelqu'un, à la première personne. La même image renvoie donc à deux attitudes narratives opposées et il faut chercher ailleurs à quel niveau s'élabore la narration filmique.

Pour définir le rapport du narrateur à l'histoire qu'il raconte, dit Genette, "la vraie question est de savoir s'il a ou non l'occasion d'employer la première personne pour désigner l'un de ses personnages ${ }^{6}$ ». Comment l'image, dans l'impossibilité sémiologique de signifier la personne grammaticale, et privée de critères aussi repérables que ceux de la langue, peut-elle signifier une attitude narrative ? Ce problème ne sera résolu qu'à partir du moment où l'on sera capable d'expliciter en quel sens on peut parler de récit au sujet d'une suite d'images mouvantes et muettes. La démarche paraît hyperbolique : comment mettre en doute que l'image raconte? Pourtant, à y regarder de plus près, cette évidence, que l'on tenait pour une donnée première, se précise, se nuance, se ramifie, faisant éclater du même coup l'idée d'une narration unitaire subsumable sous un concept unique.

Stricto sensu, le récit suppose toujours une transformation. Dans cette mesure, seul le "récit d'événement " mérite vraiment son nom puisqu'il est " transcription du non-verbal en verbal ". En revanche, le " récit de parole ", parce qu'il imite un dialogue qui est présumé réel, tend à s'identifier à la réalité au point de faire disparaître l'activité propre de celui qui raconte. "Lorsque Marcel, à la dernière page de Sodome et Gomorrhe, déclare à sa mère: "Il faut absolument que j'épouse Albertine ", il n'y a pas, entre l'énoncé présent dans le texte et la phrase cernsément prononcée par le héros, d'autres différences que celles qui tiennent au passage de l'oral à l'écrit. Le narrateur ne raconte pas la phrase du héros, on peut à peine dire qu'il l'imite : il la recopie, et en ce sens on ne peut parler ici de récit ?. " "Récit d'événements ", la suite d'images muettes ne connaît pas la distance que suppose le passage du non-verbal au verbal. S'il est possible d'annuler toute distance entre le 
filmé et le réel, ce n'est pas seulement parce que l'analogie de l'image avec la réalité occulte les codes techniques (focale, duplication, etc.), c'est aussi parce que l'énoncé iconique manque de critères grammaticaux qui lui permettraient de signifier la différence entre transcription de la réalité et discours sur la réalité (contrairement à la littérature qui distingue, par exemple, discours rapporté et discours transposé). Dans le cas de l'image de la maison en plan fixe, l'absence de marque grammaticale de la personne provoque une hésitation sur l'acte de désignation lui-même : la "traduction "voici une maison réfère à un objet qui m'est extérieur ; voici ma maison, en revanche, est doublement déictique : en même temps que l'énoncé désigne un objet de la réalité, il renvoie au locuteur. Cette difficulté à désigner le degré de deixis d'un plan a pour conséquence narratologique une confusion possible entre focalisation et narration.

En effet, s'il m'est parfois loisible d'identifier directement le signifiant filmique à la réalité filmée (en occultant les codes techniques), je peux aussi situer la suite d'images en deçà du discours narratif, en un lieu où la caméra n'est plus un stylo mais seulement un oeil. Le récit est ainsi déchiré entre un au-delà (la transparence, la mimesis absolue) et un en deçà (le regard). Pour définir le concept de narration, il faut donc tracer la frontière entre raconter et voir. Méthodologiquement, il n'est pas possible d'assimiler purement et simplement la question qui voit ? à ce qu'on nomme, en théorie littéraire, la focalisation. En effet, on n'a pas suffisamment remarqué que ce concept recouvre deux réalités narratives distinctes: d'une part, le savoir du narrateur par rapport à ses personnages (en sait-il plus, moins ou autant qu'eux?) ; d'autre part, sa localisation par rapport aux événements qu'il raconte.

Sans doute parce que faire un film, c'est d'abord assigner une place à la caméra et mettre en scène, les théoriciens du cinéma ne retiennent du terme de focalisation que la seconde acception ${ }^{8}$. C'est là une restriction illégitime : le champ romanesque montre que, dès lors que l'on prend en compte ces deux niveaux, il est rare qu'une focalisation interne, par exemple, soit totale : ainsi, le narrateur centrera le récit sur les pensées du héros sans pour autant le faire complètement disparaître de la description ${ }^{9}$. Le regard ne pouvant être rendu que par transcription verbale, il arrive que l'hiatus entre ce qui est su et ce qui est vu disparaisse de telle sorte que voir s'identifie à savoir. Aussi, quand je dis "James Bond semble étonné ", j'avoue que j'ignore ce qui se passe dans la tête du héros et, en même temps, je rends compte de la perception supposée de sa physionomie. En revanche, le cinéma travaille sur deux registres : il peut montrer ce que voit le personnage et dire ce qu'il pense. Si l'on ne veut pas restreindre, ure fois de plus, l'analyse du film à l'étude de l'image, il importe donc de différencier l'attitude narrative par rapport au personnage-héros en fonction des informations verbales et visuelles. Afin de ne pas multiplier les néologismes, je conserverai le 


\section{François Jost}

terme de focalisation pour désigner ce que sait un personnage (malgré l'ambiguïté de ce terme qui, en matière de cinéma, connote le choix de la focale). Pour caractériser la relation entre ce que la caméra montre et ce que le héros est censé voir, je propose de parler d'ocularisation : ce terme a en effet l'avantage d'évoquer l'oculaire et l'oeil qui y regarde le champ que va " prendre » la caméra. Quand celle-ci semblera être à la place de l'œil du personnage on parlera $d$ ' " ocularisation interne "; lorsque, à l'inverse, elle semblera être placée en dehors de lui, j'utiliserai provisoirement - on verra pourquoi - l'expression "ocularisation externe ${ }^{10}$.

Il est bien sûr des cas où les énoncés iconiques renvoient par leur structure à l'existence d'un regard.

Cinq critères permettent $d^{\prime}$ identifier des images comme subjectives ${ }^{11}$ :

a) L'exagération du premier plan. qui suggère la proximité d'un objectif-ceil ; par exemple, lorsqu'on voit le décor d'une chambre à travers un cache en forme de trou de serrure. ou un flou très marqué.

b) L'abaissement du point de prise de vue en dessous du niveau des yeux : photo à ras de terre (comme dans la campagne publicitaire pour la RATP où l'on voit un homme, réduit à sa paire de tennis, monter dans un autobus).

c) La représentation d'une partie d'un corps en premier plan, supposant l'ancrage dans un regard: vue d'un paysage qui défile à travers une mèche de cheveux (dans les Enchaînés, 1946); mains entrant par le bas d'une photo pour suggérer la présence hors cadre d'un homme qui allume une cigarette (publicité Gitanes).

d) L'ombre du photographe.

e) La matérialisation, dans la photo, du viseur de l'appareil.

En littérature, on reconnaît une focalisation interne lorsqu'on peut " réécrire un segment narratif sans que cette opération entraîne aucune autre altération du discours que le changement de pronoms grammaticaux : ainsi, une phrase telle que "James Bond aperçut un homme d'une cinquantaine d'années. d'allure encore jeune ", etc., est traduisible en première personne (" $\mathrm{j}$ 'aperçus", etc.), et relève donc pour nous de la focalisation interne. Au contraire, une phrase comme "Le tintement contre la glace sembla donner à Bond une brusque inspiration" est intraduisible en première personne sans incongruité sémantique évidente ${ }^{12}$. Hormis les cas que nous venons de signaler, l'image fixe ne possède pas de critères didentification aussi sûrs. Toute ocularisation externe peut être transformée en ocularisation interne sans difficulté : la photo d'un personnage qui regarde de profil dans le lointain sera aussi bien une ocularisation externe sur ce personnage qu'une ocularisation interne ${ }^{13}$ sur l'operator ${ }^{14}$. Seul l'ancrage verbal permettra de dire si la photographie n'a d'intérêt que pour le regard de l'operator ou, au contraire, pour l'objet ou la personne représenté. Les revues de photos 
répartissent d'ailleurs généralement leurs dossiers selon ces deux axes. Selon que le texte mettra l'accent sur la fonction référentielle ou sur la fonction expressive, la même photo prendra une signification différente quant à l'ocularisation (externe dans le premier cas, interne dans le second). Le plus souvent, la photo ne connaît donc qu'un mode d'ocularisation indifférencié, une sorte d'ocularisation zéro. Cette " indifférenciation " tient d'abord au fait que toute notion de point de vue n'est compréhensible que par rapport à l'existence d'un personnage : en effet, la focalisation ou l'ocularisation n'ont aucun sens in abstracto. La vertu opératoire de ces concepts est de décrire la relation du narrateur aux êtres dont il raconte l'histoire. Or, dans le cas de la photo, la difficulté vient du fait qu'il n'est pas possible de déterminer si les individus présents dans l'image et celui qui en est forcément absent (l'operator) font partie d'un mểme univers diégétique (on ne sait jamais si le photographe est "acteur" ou "témoin"). Comme le récit, l'ocularisation ne peut naître qu'avec la succession de photos. Le raccord est le seul critère susceptible de déterminer sans risque d'erreur l'ocularisation d'une image fixe. N'importe quelle photo prise sous un angle peu marqué ${ }^{15}$ peut devenir une représentation subjective si on la juxtapose à l'image d'un visage sur lequel se lit un regard. C'est cette règle qui permet au cinéma de faire passer pour vrais des raccords faux par rapport à l'espace du tournage.

Le problème de la signification sémiologique d'un plan ne peut donc être traité complètement qu'à condition d'être resitué dans le champ du récit, c'est-à-dire dans un contexte où l'on suppose quelqu'un qui raconte (un narrateur) et quelqu'un dont on raconte l'histoire (un personnage) : sans cette opération, l'analyse de l'image touche très vite le bout d'une impasse. Dans l'incapacité de résoudre le problème de la signification par rapport à l'énoncé iconique. la sémiologie doit, d'emblée, élargir son domaine d'application au "texte " visuel. Ainsi, le plan extrait d'un champ/contrechamp, bien qu'il soit l'expression du regard d'un personnage, sera aussi bien une ocularisation externe tant qu'il sera vu en dehors de son contexte. La caméra ne peut être dite "subjective» que lorsque se marque dans le signifiant la matérialité d'un corps : aux critères que nous avons répertoriés pour la photo s'ajoute, pour le cinéma, une certaine qualité de mouvement de l'opérateur, un "tremblé », un "bougé ", qui, immanquablement, renvoie à celui qui prend le film, qui regarde la scène ${ }^{16}$.

"Celui qui prend le film ", "celui qui regarde la scène »: on sent bien que ces deux expressions ne sont pas synonymes. L'une renvoie plus ou moins à ce que Metz nomme le "point de vue de l'auteur ", l'autre au "point de vue du personnage " ${ }^{1 ?}$. Il propose aussi d'opposer l'identification cinématographique primaire (= identification fondamentale à la caméra) à l'identification cinématographique secondaire qui prend " en bloc " les différents niveaux d'identification au personnage. 
D'un point de vue narratologique, il faut souligner que le régime d'identification n'est pas inscrit sémiologiquement dans le film d'une façon irréfragable. Ainsi peuvent se produire des balancements entre identifications cinématographiques primaire et secondaire. A titre d'exemple, lisons l'analyse de $M$ le Maudit (1931) par Noël Burch : "Signalons une substructure intéressante : l'organisation de la " distance" (mentale) de Frau Beckmann par rapport au champ de la caméra ; "réellement" off, c'est-à-dire hors de portée acoustique du champ, pour le plan de l'escalier, moins vraisemblablement pour celui du grenier (rien n"indique qu'elle aurait quitté son palier), " réellement ", à nouveau, pour la chaise vide, elle l'est tout à fait artificiellement pour les plans de ballons, situés manifestement à des kilomètres de distance ${ }^{18}$."

En fait, la difficulté, pour Burch, c'est de faire basculer l'identification primaire en secondaire, en l'occurrence, de justifier l'ancrage de la caméra au regard de Frau Beckmann. Cette démarche ne s'effectue pas sans mal.

En effet, une lecture précise des mouvements et des angles de caméra montrerait, à l'inverse, que les plans de la petite fille renvoient à un regard "en creux", celui du tueur (dont l'aboutissement est ce travelling, à hauteur d'homme, qui suit la fillette dans la rue pour découvrir enfin l'ombre projetée de $M$ sur l'avis de recherche apposé par la police).

Ce qu'atteste cette microanalyse, c'est que l'ancrage de la caméra à un personnage déterminé (identification secondaire) n'est pas toujours facile; mais il y a plus : la notion d'identification primaire, incontestable au point de vue sémiologique (le spectateur est toujours, dans l'idéal ${ }^{19}$, à la place de la caméra), est-elle toujours pertinente $d$ 'un point de vue narratologique?

Lorsque la caméra se contente de suivre un personnage qui marche, par un travelling ou un panoramique qui épouse son mouvement, l'identification à la caméra joue encore, certes, mais aucun rôle diégétique ne lui est conféré. D'un point de vue narratif, la place de la caméra n'est pas pertinente : c'est pourquoi, plutôt que de parler d'ocularisation externe, nous préférerons le terme d' ocularisation zéro ${ }^{20}$ ". A l'inverse, si le même mouvement de caméra est suivi du plan d'une femme qui tourne la tête, le spectateur s'identifie ponctuellement au regard du personnage : il y a ocularisation interne. Si, en revanche, la caméra tremble fortement lorsqu'un journaliste rend compte de l'entrée d'un ministre à l'Élysée, l'identification primaire prendra toute sa valeur : je serai renvoyé aux difficultés de tournage. Dans d'autres cas encore, le spectateur sent bien que la position de la caméra ne renvoie pas à un personnage et que, pourtant, son angle est marqué. Le cas le plus célèbre étant celui des fameuses contre-plongées de Welles qu'on ne peut pas toujours accrocher à un regard, mais qui ne sont pas pour 
autant insignifiantes du point de vue stylistique ou thématique. Par exemple, le fait que la fille du héros est vue à plusieurs reprises à travers un grillage, dans Monsieur Arkadin, n'est pas toujours justifié par un raccord de regard, mais, tout le film développant par ailleurs l'idée qu'elle est surveillée et espionnée par son père, cette ocularisation, même si elle n'est pas directement ancrée, est bien différente d'une prise de vue neutre, d'une ocularisation zéro.

Le concept narratologique d'ocularisation ne recouvre pas purement et simplement la réalité sémiologique du cinéma: l'identification primaire, qui est une condition perceptive, peut être diffractée et recevoir ou non une pertinence d'un point de vue narratif. Dans cette perspective, il n'est nullement contradictoire de parler d'ocularisation zéro lorsque la caméra ne vaut pas pour une instance diégétique à l'œuvre dans la fiction, alors même que l'appareil de prise de vues est nécessaire. Cet ancrage ou ce non-ancrage de la caméra à une instance narrative explique d'ailleurs des différences sociologiques de perception : le film de reportage sera tantôt ressenti comme la transcription de la réalité à l'état brut (= ocularisation zéro), tantôt comme un regard subjectif ( = ocularisation interne) renvoyant à la personnalité du reporter. La caractérisation sémiologique est donc interprétée en fonction d'une autre pertinence.

Jusqu'à présent, on a envisagé la focalisation par rapport à l'ordre proche que constitue le montage. De ce point de vue (si l'on peut dire !), elle apparaît comme essentiellement différente de la focalisation romanesque : si celle-ci, parce qu'elle est la transformation d'un événement en verbalisation, appartient toujours à l'ordre du raconté, c'est-à-dire du transposé, la focalisation cinématographique (l'ocularisation) pourra quitter le domaine du récit pour rendre d'emblée la perception visuelle : comme on vient de le dire, n'importe quelle image succédant à un plan mettant en scène un personnage qui regarde. sera sentie comme appartenant à son paysage visuel. Parce qu'elle est directement du côté du voir, l'ocularisation peut donc être considérée comme en deçà du récit.

\section{L'acte narratif : voyage dans l'au-delà.}

$\mathrm{Si}$, comme on l'a souvent dit, l'image mouvante ne connaît qu'un seul temps, le présent, il ne faut pas s'étonner qu'on dénie au cinéma le droit d'être un récit à part entière. En effet, devant la quasi-impossibilité d'introduire une distance entre l'activité racontante et le raconté, l'image semble passer par-delà le récit pour atteindre directement le réel. Derrière le plaidoyer de Bazin contre le montage ${ }^{21}$, il n'y a pas seulement une conception presque mystique de la réalité, il y a aussi 


\section{François Jost}

l'illusion (l'incompréhension) que le cinéma peut parler du monde sans être récit. Contrairement à la littérature, qui est susceptible d'introduire une distance entre temps de la diégèse et temps de la narration. le plan-séquence muet paraît condamné à ce que Genette nomme la "narration simultanée » : lorsque je vois un long plan montrant Jeanne Moreau, de face, qui prépare une omelette (dans Souvenirs d'en France d'André Téchiné, 1974), diégèse et récit se confondent : ce qui est montré est aussi ce qui est dit (mais par qui ?), de sorte qu il m'est très difficile de repérer une instance narrative.

Ce qui caractérise un récit littéraire au présent. c'est qu'il peut fonctionner de deux façons opposées, selon que l'on met l'accent sur l'histoire ou sur le discours narratif ${ }^{22}$. Il en va de même au cinéma : lorsque la façon de filmer est dictée par la représentation, les codes analogiques entraînent l'image vers la mimesis, et le récit en tant que tel n'est plus perçu : ainsi, un panoramique suivant un personnage ou un léger recadrage correspondant à un geste basculeront du côté de l'histoire. En revanche, un plan décadré, un travelling au mouvement mécanique mettront en évidence la présence d'un narrateur intervenant sur la réalité filmée. Dans le premier cas, l'histoire semble se raconter toute seule; la narration est réduite à l'ocularisation zéro : le cinéaste s'avance masqué. Dans la mesure où les événements sont vus par une personne sans existence dans l'image, on peut dire qu'on est en présence d'une sorte de narration visuelle à la troisième personne. Toutefois, c'est là une étiquette toute théorique qui doit mal correspondre à la réalité sociologique : nul doute que le film de reportage soit reçu dans certaines catégories sociales comme la réalité à l'état brut, sans manipulation d'une tierce personne. De même, certains films historiques suscitent des réactions au premier degré (cf. Molière d'Ariane Mnouchkine, 1978) qui sont au-delà de la perception de tout acte narratif : l'image entraîne directement des jugements sur la conformité au référent (du type "les gens étaient drôlement habillés ". "il y avait du monde dans les rues, à l'époque", etc.). En fait, l'acte narratif n'est réellement sensible qu'au moment où l'image se détache de l'illusion mimétique (par décadrage, mouvement étrange de la caméra, raccord, etc.), c'est-à-dire lorsque, à travers les énoncés visuels, sont perçues des marques d'énonciation. C'est parce que jai soudain la conviction " qu'on me parle cinéma " que je prends aussi conscience que quelqu'un est en train de me raconter une histoire. Dans la mesure où tout se passe comme si quelqu'un affirmait tout à coup sa "subjectivité " dans le langage cinématographique, je peux identifier ce mode de narration au discours dans la langue et donc parler métaphoriquement d'une narration visuelle à la première personne. Le narrateur est alors plus ou moins identifié à celui qui possède le pouvoir de faire et d'agencer les images : un "grand imagier " selon le mot de Metz. Il faut toutefois nuancer la distinction que nous venons d'établir par les remarques suivantes : 
- toute "narration" constituée d'une suite d'images muettes est aussi un parti pris de regard sur l'événement raconté, une ocularisation. Il faudrait donc un terme qui rende compte de l'équivalence suivante : parler cinéma, c'est voir et montrer des images ;

- s'il est possible d'établir une distinction théorique entre une narration à la première personne et une narration à la troisième personne, il ne faut pas oublier non plus que le langage cinématographique ne possède pas véritablement de signes énonciatifs ${ }^{23}$. Le mểme récit pourra donc être tiré d'un côté ou de l'autre selon le public. et le même film, perçu comme transparent ou, au contraire, proprement cinématographique. Le degré de présence du narrateur est en effet proportionnel à l'idée que l'on se fait de la mimesis : nul doute que des arrêts sur l'image (en cours de mouvements comme dans Sauve qui peut (la vie), 1980) renverront d'emblée à un "grand imagier "(plus ou moins assimilé à Godard); en revanche, la focale employée pour un plan fixe sera moins facilement perçue comme un acte narratif : tout dépendra de la commutation mentale effectuée par le spectateur avec une autre focale possible en ce point de la chaîne filmique. Dans le cas d'une suite d'images muettes, le narrateur est plus une construction du spectateur qu'une instance repérable grâce à des marques immanquables. Cela revient à dire que l'identification du spectateur à ce qui se passe sur l'écran est souvent incertaine et flottante : en effet, d'une part, la prise de conscience du dispositif est plus ou moins grande selon les individus ("identification cinématographique primaire ", Metz), mais, d'autre part, dans certains cas, le statut de l'image lui-même est ambigu : lorsqu'un film est inauguré par un long travelling, par exemple, il ne m'est pas toujours facile de déterminer si j'épouse le discours-regard du cinéaste ou si je vois à travers les yeux d'un personnage ("identification cinématographique secondaire ${ }^{24}$ »). (Je pense en écrivant ces mots à certains passages de Moderato cantabile de Peter Brook, 1960.) En généralisant ce type de doute, le cinéma moderne rend complexe le choix d'un niveau d'identification. Est-ce parce qu'il refuse au destinataire la possibilité de se structurer une identité spectatorielle qu'il provoque de nombreux rejets?

Comme on l'a dit supra, la photographie renforce cette hésitation du lecteur; suis-je à la place de loperator (identification primaire) ou suis-je un personnage-spectator (identification secondaire)? Nul doute que la réponse à cette question explique en partie l'attirance ou la répulsion du public pour certaines images ${ }^{25}$.

C'est seulement lorsqu'un acteur parle face à la caméra que la présence d'un narrateur est révélée de façon irréfragable. Même dépourvue de son, une telle mise en scène accrédite l'idée qu'on me raconte une histoire, à condition que le plan ne soit pas raccordé au précédent par un simple champ/contrechamp, ce qui le transformerait en ocularisation interne. Une seule condition nécessaire pour identifier le 


\section{François Jost}

narrateur : que ses yeux regardent l'objectif de la caméra ${ }^{26}$, vers moi donc; alors je transformerai l'énoncé " il raconte " en «il me raconte ». Sans ce regard frontal, j'aurais aussi bien l'impression d'assister à un dialogue, forme mimétique par excellence, au-delà du récit. Affichant son rốle dans le récit, ce type de narrateur est avant tout un conteur. Si la présence d'un narrateur en dehors du profilmique est révélée par le langage cinématographique lui-même (du moins, tant que l'on raisonne dans le cadre du cinéma muet), le narrateur profilmique affirme d'emblée son statut en installant le spectateur dans le circuit de la communication filmique. L'acte narratif n'est compris comme tel que parce qu'il s'adresse à moi : si l'on me parle, c'est manifestement pour me raconter. Lorsque le narratcur n'est pas un personnage attesté par l'image, son identification est largement variable selon le spectateur, puisqu'elle dépend de l'idée que celui-ci se fait du langage cinématographique et de sa faculté à être mimétique; en revanche, lorsque le narrateur profilmique s'adresse à la caméra, sinstaure un dispositif dans lequel je ne peux pas ne pas entrer. Toutefois, sans l'aide du son ou, au moins, de mentions écrites, il m'est encore impossible de déterminer la relation de celui qui parle à la diégèse. Le problème de la narration filmique ne se pose donc pas de façon similaire selon que le narrateur est absent de l'image ou qu'il est intégré au profilmique : $d u$ "grand imagier " au "conteur ", l'énonciation se déplace du langage cinématographique au récit filmique. Dans ce second cas, je comprends la présence d'une instance narrative indépendamment de ce qu'elle dit, alors que, dans le premier cas, je ne la soupçonne que parce qu'elle dit ou montre d'une certaine manière. Attentif aux gestes d'un grand imagier, je prends seulement conscience d'assister à un récit : on me raconte, ce n'est donc pas la réalité même; le conteur, quant à lui, me situe à l'intérieur d'un récit, dévoilant d'emblée sa relation à la diégèse. Après avoir délimité les frontières théoriques de la narration à travers les problèmes du langage visuel. il convient maintenant d'envisager le fonctionnement de ce concept dans le film, en relation avec les informations verbales ou écrites.

\section{LA NARRATION POL YPHONIQLE.}

Nous nous limiterons, dans le cadre de cet article, au narrateur extradiégétique, c'est-à-dire à ce personnage qui, explicitement, est l'instance productive du récit. Au cinéma, ce type de narration connaît au moins trois cas de figures : la voix in, le carton, et la voix off. 
In : le conteur.

On ne saurait juger de la narration verbale sans examiner simultanément comment elle est représentée : en effet, prenons le cas d'un "conteur» qui dit une histoire qui lui est arrivée à lui-même ou à d'autres. Réduisons sa mise en scène à celle que l'on impose aux présentateurs télé (comme dans les émissions d'Alain Decaux ou de Pierre Bellemare); assis devant un fond indifférencié, il raconte. Manifestement, il est maître des événements diégétiques : qu'il les narre en focalisation externe, interne ou zéro (selon les catégories de Genette), il semble responsable du récit. Et pourtant : quand bien même il dira * je », il sera toujours sous le regard de la caméra, en ocularisation zéro. Quelle que soit sa position par rapport à la diégèse, il dépend d'une autre instance qui, à défaut de la raconter, le montre. On peut bien sûr supposer que ce personnage absent qui dirige la caméra vers le conteur est le conteur lui-même (il est alors acteur et metteur en scène). Peu importe; il s'agit moins d'un problème de personne que d'un problème de niveau : tant que l'on reste en plan fixe (comme le fait la vidéo "directe"), la situation énonciative est quasi transparente et le " conteur " est un narrateur "extradiégétique ". Mais il suffira de la moindre avancée de caméra, de la moindre coupure à l'intérieur du plan, pour que la narration glisse et change de niveau : soumis au bon vouloir d'un grand imagier, le conteur deviendra lui-même l'un des éléments de la diégèse ; d'extradiégétique, il rétrogradera alors au statut d'intradiégétique ${ }^{27}$. Ce changement de niveau sera plus ou moins bien perçu selon la sensibilité à l'énonciation cinématographique et, donc, selon l'idée que l'on se fait de la mimésis. Cette mobilité, toujours possible, de l'extra- vers l'intradiégétique est extrêmement fréquente dans les actualités télévisées : un dirigeant syndical ou un ministre parle à la télévision face à la caméra, les mots s'enchaînent " naturellement " et l'on oublie presque le petit écran pour plonger dans la réalité ; soudain l'image saute, révélant une coupure effectuée au montage : alors, à l'insu du premier narrateur, se révèle une autre instance plus puissante, dont le nom change selon les cas: journaliste, chef de l'information, censeur...

\section{Carton-Voix off.}

Jusqu'à un certain point, il est possible de traiter parallèlement la narration par carton ou par voix off dans la mesure où ces deux modes ont en commun de gommer la représentation du narrateur. Toutefois, il faut les différencier sur l'axe de la simultanéité : tandis que la voix off peut se poursuivre sur l'image, le carton intervient toujours ponctuellement. 
Le narrateur extradiégétique peut être profilmique, c'est-à-dire présent et identifiable comme personnage dans la représentation visuelle de l'histoire qu'il raconte, ou extraprofilmique (absent de la représentation visuelle de l'histoire qu'il raconte). Genette a proposé de nommer ces deux types d'attitude par rapport à la diégèse homodiégétique et hétérodiégétique. Dès lors, on peut schématiser les relations de la narration dite ou écrite à la narration visuelle de la façon suivante :

\begin{tabular}{|l|l|l|}
\hline $\begin{array}{c}\text { Niveau de la } \\
\text { narration } \\
\text { (induit du verbal) }\end{array}$ & \multicolumn{1}{|c|}{$\begin{array}{c}\text { Relation à la } \\
\text { diégèse }\end{array}$} & \multicolumn{1}{|c|}{$\begin{array}{c}\text { Relation du narrateur } \\
\text { à l'image }\end{array}$} \\
\hline \multirow{5}{*}{ Extradiégétique } & Homodiégétique & $\begin{array}{l}\text { présent comme person- } \\
\text { nage : profilmique } \\
\text { (ocularisation zéro) }\end{array}$ \\
\cline { 2 - 2 } & $\begin{array}{l}\text { Hétérodiégétique } \\
\text { (raconte une his- } \\
\text { toire dont il est } \\
\text { absent) }\end{array}$ & $\begin{array}{l}\text { absent de l'image } \\
\text { (ocularisation interne) } \\
\text { (ocularisation interne) }\end{array}$ \\
\hline
\end{tabular}

Il va de soi que le narrateur textuel (qu'il apparaisse par la voix ou dans les mentions écrites) peut s'analyser au moyen des concepts de la théorie littéraire. En particulier, son récit adopte forcément un type de focalisation (au sens que Genette assigne à ce terme). Pour que ce tableau soit complet il faudrait donc ajouter une colonne concernant cette catégorie. On aboutirait alors à une combinatoire plus vaste où certains cas seraient possibles, d'autres probables, d'autres rares (par exemple un narrateur à la première personne, à focalisation externe et ocularisation interne, c'est-à-dire un personnage qui raconterait son histoire en "caméra subjective" tout en étant "omniscient"). Mais voyons les types de narration répertoriés par ce tableau :

\section{Homodiégétique.}

Narrateur présent comme personnage dans l'image (profilmique) :

Que la narration commence par un carton ou par une voix off à la première personne le fonctionnement est globalement identique du point de vue narratif. La différence principale entre ces deux modes de présentation tient à la façon dont s'effectue l'ancrage du texte sur le personnage visuel : dans le cas du muet, l'apparition d'une phrase à la 
première personne nécessite la succession immédiate d'un plan du personnage qui est censé raconter (soit pensif, soit en train d'écrire) ${ }^{28}$; la voix off, quant à elle, peut être " accrochée " à son propriétaire physique par des voies multiples qui mériteraient qu'on s'y arrêtât (recoupement avec la voix in, "désignation ", gros plan du personnage les yeux dans le vague, etc.). Pour simplifier, on a assimilé ici le narrateur homodiégétique profilmique à un personnage en ocularisation zéro: on peut en effet considérer que, généralement, lorsque le narrateur est dans l'image, il est vu du dehors sans appartenir pour autant au regard d'un autre personnage ${ }^{29}$. Toutefois, dans des cas extrêmes de "caméra subjective "(cf. la Dame du lac), il est envisageable que l'on montre une partie du narrateur (une main par exemple) : le personnage apparaît alors partiellement au niveau profilmique tout en étant en ocularisation interne. A l'inverse, lorsque le personnage n'est pas visible à l'écran mais qu'il raconte, off, son histoire, on pourrait admettre que la caméra ne rende pas compte de son regard (c'est toutefois un cas limite à la frontière de l'absurde : s'il adopte un point de vue externe, dans la mesure où il appartient à la diégèse, il apparaîtra plus probablement sous la forme d'un personnage profilmique). Quand le narrateur agit dans l'image comme personnage, le « je " du carton ou de la voix off se transforme en il dans l'image. En quittant la position frontale par rapport à la caméra, il quitte le dispositif énonciatif décrit supra, il abandonne le discours pour appartenir à l'histoire. Dans le cas où le narrateur ne se désigne comme tel (c'est-à-dire comme instance racontante) que par un carton ou par une présence off, on peut considérer que toute image où il apparaît comme personnage relève de son récit et donc qu'il joue le rôle de "grand imagier". L'analyse narratologique est plus complexe lorsqu'un personnage se met, à l'intérieur de l'image, en position de conteur. C'est par exemple ce qui se passe dans Cet obscur objet du désir: dans un train, un personnage commence, in, à raconter son histoire aux autres occupants du compartiment. En regard de son récit verbal, il est bien un narrateur extradiégétique, toutefois, en tant qu'il intervient lui-même dans l'image pour raconter, il appartient au discours d'un autre narrateur ${ }^{30}$ : comme le "conteur" du premier type, il peut donc à son tour rétrograder au niveau intradiégétique. En toute rigueur, on ne devrait donc donner l'appellation d'extradiégétique qu'aux narrateurs assimilables au "grand imagier", c'est-à-dire à ces voix off qui semblent organiser toute la suite des plans. On retrouve là une ambiguité qui tient à la nature double du récit filmique. Partagée entre le narrateur verbal et son récit visuel, la narration est une résultante complexe, un assemblage de poupées russes où le récit verbal englobe un récit visuel pour mieux révéler la présence d'un ultime narrateur qui agence ces pantins d'images et de sons.

Lorsque le récit verbal d'un personnage est relayé par sa représenta- 


\section{François Jost}

tion à l'image, deux solutions sont envisageables : (I) soit sa voix (off) disparaît pour laisser place à une visualisation des événements (qui pourra s'accompagner d'interventions in du narrateur-personnage); (II) soit il commente la bande-image, off, sa voix venant en contrepoint du lacis audiovisuel de la diégèse. Dans le cas où la voix du narrateur s'estompe devant le récit visuel, il est supposé qu'il en assume la responsabilité (= "grand imagier"). Dans la mesure où l"image montre sans modalisation possible ${ }^{31}$, le degré de focalisation (au sens de Genette) est largement dépendant du point de vue avoué par le narrateur.

S'il est en focalisation externe. on est alors dans cette "situation paradoxale ", dont parle Genette à propos du récit proustien, d'un narrateur à la "première personne " et pourtant omniscient. Ce paradoxe est accentué au cinéma par le fait que le personnage qui raconte son histoire est aussi vu de l'extérieur. On doit donc admettre soit qu'il se dédouble et se regarde, soit qu'il appartient au regard d'un autre, une instance d'un degré supérieur, le cinéaste. Lorsqu'il est en focalisation interne, la situation est " cohérente " en littérature, mais elle reste curieuse au cinéma puisque la personne est encore vue de l'extérieur. Rashomon offre une situation narrative particulièrement riche et complexe. Comme on sait, ce film raconte l'histoire de la mort d'un samouraï. Quatre personnages - un brigand, la femme du samouraï, l'âme du mort, un bûcheron - racontent leur version. Chacun de ces récits, dont le contenu varie considérablement (du crime au hara-kiri), est en focalisation interne : le personnage rend compte de sa façon propre de voir les choses et de ce qu'il sait sur les événements. Dans la mesure où les quatre narrateurs interviennent dans la représentation visuelle de la mort du héros, il semble que l'on soit en présence d'une ocularisation externe ou zéro et que l'on retrouve "la situation paradoxale » que nous venons de décrire. En fait, à y regarder de plus près, ce jeu sur l'opposition interne/externe se renverse une nouvelle fois, justifiant les distinctions que nous avons établies plus haut. Témoin le premier récit du bûcheron : l'homme est représenté de l'extérieur ; on le voit d'abord de face, puis de dos en plan très rapproché, avançant, sa hache sur l'épaule. Les mouvements d'appareil qui, loin de se modeler sur celui qui évolue dans l'image, suivent un parcours autonome, de même que les angles de prises de vue, trahissent la présence d'une autre instance, absente de l'image - un autre grand imagier - qui emprunte, par instants, les marques de la caméra subjective définies supra.

Si aucun personnage attesté dans la diégèse ne semble endosser la place de la caméra, cette ocularisation zéro a pourtant tendance à se confondre avec l'ocularisation interne sur une autre instance narrative, située en dehors de l'image. En jouant à la fois sur le verbal et sur le visuel et, enfin, sur les marques de langage, le film est susceptible de 
nous plonger dans des enchâssements narratifs vertigineux. Dans d'autres films, la voix off du narrateur accompagne son récit visuel. A l'intérieur du cinéma de consommation courante, son rôle est, d'une part, de combler tout ce qui manque dans l'image (ellipses spatiales et temporelles), d'autre part, d'exprimer les analyses psychologiques que le jeu de physionomie de l'acteur ne suffirait pas à exprimer. Ainsi, le personnage qui raconte son histoire peut-il exprimer de l'intérieur (focalisation interne par la voix off) ce que nous le voyons vivre de l'extérieur (ocularisation zéro) : la voix off permet donc de construire en corrélation avec l'image une narration complète puisqu'elle apporte l'intimité des sentiments à l'objectivité des événements. A pratiquer des analyses textuelles particulières, on trouverait des fonctions multiples et différenciées à ce type de narrateur; lorsqu'il parle d'une aventure passée, il est souvent témoin ou interprète, selon qu'il met l'accent sur les événements ou sur ses sentiments (cf. de nombreux films américains); dans les films modernes, il est plus souvent un spectateur ou un reporter qui commente en direct le récit que suggère la bande-image : mais les informations données en focalisation interne seront toujours sanctionnées par la contre-épreuve de l'ocularisation zéro : ainsi, si, au début de l'Homme qui ment, le héros est un simple spectateur-reporter (il décrit le paysage dans lequel il avance), son "mensonge " sera bientôt dévoilé par le décalage progressif entre ce qu'affirme le narrateur verbal et l'objectivité de l'ocularisation zéro.

Dans certains films, comme Monsieur Arkadin, le lacis audiovisuel révélera d'énormes décalages entre ce que croit savoir le héros (commentaire off en focalisation interne) et ce que le spectateur voit simultanément ou consécutivement en ocularisation zéro (ainsi von Stratten dit qu'il ne sait pas ce qu'est devenue Milly et suppose qu'elle a dû tenter sa chance, seule. I e. spectateur, lui, la voit dans un bar à Tanger, sous le regard d'Arkadin (qui l'observe à travers une vitre) : elle-même ne remarque pas cet " espionnage " dont elle est victime). Il faudrait analyser ce film en détail et montrer que l'on a les étapes suivantes: 1. le narrateur-héros en sait plus long que ce que nous voyons ; 2 . il en sait moins ; 3 . le spectateur a, seul, le savoir de tous les événements simultanés.

\section{Narrateur absent de l'image comme personnage.}

Il est rare que le narrateur présent par la voix ou par un carton soit totalement absent de la bande-image d'un film. Toutefois ce cas de figure se produit souvent ponctuellement, en particulier lorsque le plan est en caméra subjective. Tout plan fixe, même lorsqu'il ne comporte aucune marque énonciative de la première personne, peut être transformé en ocularisation interne dès lors que la voix off à la première personne le prend en charge et l'accompagne. Cette combinaison de la 


\section{François Jost}

focalisation et de l'ocularisation est en fait la plus cohérente puisque sont associés deux points de vue internes; mais le cinéma de consommation courante évite de l'utiliser sur toute la longueur d'un film, sans doute en partie parce qu'elle fait disparaître l'un des attraits du spectacle : l'acteur.

\section{Hétérodiégétique.}

Si l'on suit la lettre de Genette - et pourquoi ne la suivrait-on pas ? - ce type de narrateur ne peut intervenir dans la bande visuelle comme personnage. Le texte off pourra adopter tous les types de focalisation. Ainsi, dès le premier carton, le narrateur de la Jetée annoncera-t-il son parti pris de se placer souvent en focalisation interne. ("Ceci est l'histoire d'un homme marqué par une image d'enfance. La scène qui le troubla par sa violence, et dont il ne devrait comprendre que bien plus tard la signification... ») Ensuite. le commentaire off oscillera sans cesse entre la focalisation interne pour exprimer les pensées du héros (par exemple "il pensait se trouver en face du savant fou...") et la focalisation externe (" il vit un homme sans passion", simple description de l'image d'un médecin qui se penche sur le héros). En passant progressivement au présent, le narrateur finit par devenir une sorte de reporter qui décrit l'image ("Ils marchent. Ils s'arrêtent devant une coupe de séquoia couverte de dates historiques"). Il va de soi que le mode de narration le plus cohérent est celui qui associe focalisation interne à ocularisation interne sur le narrateur.

On a dit supra que la contre-épreuve de la focalisation interne pour un narrateur homodiégétique était l'ocularisation zéro. A l'inverse, pour le narrateur hétérodiégétique, la contradiction pourra surgir de la bouche même du personnage dont il raconte l'histoire. Par exemple, dans un film comique, le héros prendra à partie le narrateur, refusant de se voir prêter telle intention ou telle pensée (comme le font les différents personnages de la bande dessinée Kador lorsqu'ils s'adressent au dessinateur supposé). Le narrateur hétérodiégétique en voix off est assez rare. Si l'on admet assez facilement de ne rien savoir sur celui qui organise les images du film (lorsqu'il n'y a pas de narrateur attesté), il semble, en revanche, plus difficile de laisser n'importe qui franchir le seuil rassurant du monde diégétique. La narration hétérodiégétique semble réservée au documentaire : parlant pour la science ou au nom de l'objectivité de l'information, le narrateur se devra de rester anonyme, simple voix sans corps. Dès qu'il se mêle de décrire les faits et gestes d'un personnage de fiction - pour peu qu'il fasse son récit au présent - il tend à usurper la place du spectateur et à devenir le commentateur d'un film qui se fait devant lui. Ainsi India Song (1974), jouant sur focalisation et ocularisation. bâtit une narration paradoxale : des voix 
sans identité, résolument hétérodiégétiques, viennent commenter le déroulement progressif de la série visuelle :

(La caméra remonte le long du corps de Lol V. Stein)

V 1 : Il l'avait suivie aux Indes.

V 2: Oui... Pour elle il avait tout quitté. En une nuit.

V 1: La nuit du bal?

V 2: Oui...

V 1: Michael Richardson était fiancé à une jeune fille de S. Tahla, Lol Valérie Stein...

V 2: Le mariage devait avoir lieu à l'automne. Puis il y a eu ce bal... ce bal de S. Tahla.

V 1 : Elle était arrivée tard à ce bal... Au milieu de la nuit... habillée de noir...

Que d'amour, ce bal, que de désir.

Ces voix en savent souvent plus que nous sur les personnages que nous voyons évoluer sur l'écran, elles leur apportent un passé ; par ailleurs, elles éprouvent presque de l'intérieur ce qu'ils sont en train de vivre. La narration hésite donc continuellement entre focalisations externe et interne. Pourtant ce récit dialogué n'avance qu'en fonction de l'image. Ces narrateurs hétérodiégétiques, ces témoins invisibles, ont le même point de vue que nous, spectateurs : ce que nous voyons, c'est d'abord ce qu'ils regardent, de sorte que l'on peut considérer à bon droit que nous sommes en ocularisation interne et que nous n'assistons qu'à ce qu'ils mettent en image : les personnages s'identifient au "dispositif " cinématographique, et identifications cinématographiques primaire et secondaire finissent par se confondre.

Narration, focalisation, ocularisation... Chacun de ces niveaux joue de façon polyphonique à l'intérieur de tout film. A n'en pas douter, des analyses textuelles, plus fines que celles que l'on a suggérées ici, révéleraient que l'autonomie relative des strates narratives existe même à l'intérieur des films de consommation courante.

Par ce jeu entre le dit et le montré, le cinéma met le spectateur dans une position très particulière par rapport au savoir narratif : déchiré entre ce qu'on lui apprend (par la voix) et ce qu'il découvre (de ses propres yeux), ne suit-il pas, à nouveau, le parcours initiatique de l'enfant qu'il a été ?

François Jost

Université de Paris III 


\section{NOTES}

1. La narration sera ici entendue au sens strict, que lui donne Genette. d" "acte narratif producteur $\gg$.

2. Dans « Le Je à la recherche de son identité ", in Poétique, n" 24, Paris, Éd. du Seuil, 1975, j'ai tenté de montrer comment l'utilisation de quelques déictiques révélait la présence du narrateur à l'intérieur de grands pans $d$ ' " histoire ", au sens de Benveniste.

3. Cf. * Frontières du récit*, in Figures II, Paris. Éd. du Seuil. 1969. dans lequel Gérard Genette montre que le texte de Balzac cité par Benveniste comme archétype de "l'histoire » contient, en fait, plusieurs passages qui, manifestement, appartiennent au " discours *.

4. Sur ce point. on se reportera aux schémas proposés par Mitry, Esthétique et Psychologie du cinéma. t. II Paris, Éd. universitaires, 196.5.

5. Metz, Essais sur la signification au cinéma, t. I, Paris, 1971, p. 118. Il faut toutefois noter que, en même temps qu'il soulignait la ressemblance de l'image et de l'énoncé, Metz mettait en garde contre une réduction hâtive de l'une à l'autre : "Le plan filmique ressemble donc à un énoncé plutôt qu'à un mot. Cependant il serait faux de dire que le plan équivaut à un énoncé.

6. Gérard Genette, Figures III, Éd. du Seuil, 1972, p. 252.

7. Ibid., p. 190.

8. Cf., par exemple, Vanoye, Récit écrit, Récit filmique, Paris, Cedic, 1969, p. 15 ? : * La focalisation zéro : plans généraux vus du point de vue de Sirius (ou de Dieu, si l'on préfère : mais pourquoi rapporter à une conscience ce qui n'est qu'une localisation?) » (Mot souligné par moi.) M. Lagny, M.-C. Ropars et P. Sorlin, "Analyse d'un ensemble extensible », Théorie du film, Paris. Albatros, 1980, p. 135 : “Cette catégorie reprend la question proprement narratologique de savoir quel foyer de perception est choisi pour médiatiser la représentation. *

9. Cf. Gérard Genette, Figures III, p. 209 : "Il faut aussi noter que ce que nous appelons focalisation interne est rarement appliqué de façon tout à fait rigoureuse. En effet, le principe même de ce mode narratif implique en toute rigueur que le personnage focal ne soit jamais décrit, ni même désigné de l'extérieur. *

10. La segmentation du concept de focalisation devrait être poussée plus loin. Si, en effet, on peut caractériser la relation du personnage à ce que montre la caméra, il semble tout autant nécessaire d'envisager la bande-son par rapport au même personnage. Il est bien évident que l'occurrence d'un bruit ou d'un dialogue, comme le mixage lui-même. peut s'ancrer de façon plus ou moins subjective dans la fiction. Par exemple, lorsque, sur l'image d'un personnage qui ne parle pas, on entend une bribe de texte ou un bruit que le contexte spatial ne justifie pas, bien souvent il faut interpréter cette intervention sonore comme un souvenir ou une *imagination " de ce personnage. Il existe des cas plus complexes où le mixage crée de véritables points de vue sonores : qu'on se souvienne de la Pointe courte ou de l'Immortelle (Alain Robbe-Grillet. 1963) où l'éloignement des acteurs dans l'écran va à l'encontre de la permanence du "niveau " des dialogues.

Ce domaine du * point de vue " sonore mériterait une étude autonome qu'il me faut remettre à plus tard. Par symétrie avec le concept d'ocularisation, je proposerai le terme d'auricularisation.

11. Cette liste, qui s'applique à l'image fixe, n'est sans doute pas exhaustive.

12. Genette, Figures III, p. 210.

13. Récemment. un fabricant de pellicules et d'appareils photographiques, voulant faire une publicité, avait choisi une photographie d'un bord de mer exotique. Cette image était lue, dans la plupart des cas, comme une référence au pays lui-même, et donc: 


\section{Narration(s) : en deçà et au-delà}

rattachée à des campagnes pour clubs de vacances. Il a fallu rajouter sur cette image le dessin d'un viseur pour mettre l'accent sur l'acte photographique.

14. Terme emprunté à Barthes (la Chambre claire).

15. Au sens où Lotman définit un élément * marqué * comme * la transgression d'une attente, (Esthétique et Sémiotique du cinéma, Paris, Éd. Sociales, 1977, p. 59).

16. J'ai développé ces remarques dans * Discours cinématographique, narration : deux façons d'envisager le problème de l'énonciation ", in Théorie du film.

17. Le Signifiant imaginaire, Paris, UGE, 1977, coll. * 10/18 *, p. 77.

18. * De Mabuse à $M$ *, in Revue d'esthétique, 1973-2.3.4, Klincksieck.

19. Dans la pratique, sa perception est bien loin d'occuper cette place idéale.

20. Cette appellation n'est nullement un jugement péjoratif : j'insiste sur le fait qu'elle signifie seulement que la caméra ne prend pas la place d'un personnage du récit. Si l'expression * ocularisation externe " me paraît difficile à conserver, c'est que, stricto sensu, elle est contradictoire : si on peut localiser un oil (condition sine qua non pour parler d'ocularisation) on doit aussi pouvoir l'ancrer. Le fait que sa localisation n'est pas pertinente du point de vue narratif ne revient pas à confondre toutes ocularisations zéro. D'autres critères (stylistique, rhétorique, etc.) peuvent, par ailleurs, être pris en compte.

A la limite, le terme d'ocularisation externe pourrait caractériser les cas de dédoublement dans lesquels un personnage semble se voir de l'extérieur (cf., par exemple, certains moments de l'Immortelle).

21. Montage interdit ", in Qu'est-ce que le cinéma? t. I, Paris, Éd. du Cerf, 1958.

22. Gérard Genette, Figures III, p. 231.

23. Cf. Discours cinématographique, Narration..., op. cit.

24. Le Signifiant imaginaire, p. 79.

25. Les notions de voyeurisme et d'exhibitionnisme devraient être rapportées à ces considérations strictement sémiologiques. Afin de sauvegarder l'unité de ma problématique, je remets ce développement à plus tard. Je me bornerai à ces quelques remarques : la différence entre les jugements concernant l'érotisme ou la pornographie d'une photographie tiennent généralement au fait que, dans le premier cas, je m'identifie au photographe, dont j'apprécie le travail sur la réalité (d'où le succès public de David Hamilton), et, dans le second, je me sens violemment mis en situation de voyeur, seul, face à une réalité sans médiation : je suis un personnage qui regarde et non plus celıi qui juge le regard d'un autre.

26. Péninou, dans Intelligence de la publicité (Paris, Laffont, 1972), a noté que c'est la direction du regard, de même, qui rend un message publicitaire implicatif : Tout personnage qui regarde n'est pas nécessairement regardant du lecteur, un code de position va donc jouer : il est donc nécessaire que le regard soit droit, les yeux grands ouverts tournés délibérement vers le lecteur de la page * (p. 189).

27. J.-P. Simon me fait remarquer que le terme intradiégétique est peut-être trop vite avancé. En effet, ma formulation sous-entend que la série visuelle du conteur a le statut d'un événement raconté, et donc qu'il fait partie de la diégèse. Le problème est complexe parce que le film parlant met en jeu deux narrations (comme on y reviendra plus loin) : l'une visuelle, l'autre verbale ; la difficulté est de les hiérarchiser. J'admets ici que les effets discursifs à l'intérieur de la bande-image rétroagissent sur la narration verbale et tendent à transformer le récit d'un événement en un événement d'un autre récit.

28. Je n'ai pas d'exemple en tête au moment où j'écris ces lignes, mais nul doute que cette possibilité théorique soit attestée dans l'histoire du cinéma.

29. Si ce n'est ponctuellement dans les champs/contrechamps. Dans certains cas, il peut toutefois arriver que le narrateur, qui croit maîtriser les événements, soit observé à son insu et, donc, en ocularisation interne sur un autre personnage.

30. Ce phénomène se retrouve dans un film muet comme Caligari. Au début, Franz, assis sur un banc, dans un asile, raconte son histoire à un compagnon : un récit visuel 
vient relayer les premiers cartons (* Le drame que nous avons vécu est à peine crovable. Je vais vous en raconter l'histoire. ") A la fin du film. le retour au présent est l'occasion d'une scène dont Franz n'est plus narrateur (verbal) et dont le témoin est le directeur de l'asile qui s'adresse au public en disant : "Maintenant jai compris. Il me prend pour Caligari. Je crois que je vais pouvoir le guérir. "

31. Rappelons qu'on appelle "termes modalisants ", " les termes comme peut-être. certainement, sans doute (qui) suspendent l'assertion du sujet énonçant et, par là. se rattachent à l'énonciation ". Todorov-Ducrot. Dictionnaire encyclopédique des sciences du langage, Paris, Éd. du Seuil. 1972. p. 106. 